\section{Hier steht eine Anzeige.}

Springer
In beiden Studien schnitten zwar die jüngeren Frauen bei der brustkrebsfreien 5-Jahres-Rate mit insgesamt nur 79\% deutlich schlechter ab. Allerdings erhöhte sich in der SOFT-Studie der Anteil von $75,9 \%$ unter Tamoxifen plus OFS bzw. $67,1 \%$ bei alleiniger Tamoxifen-Therapie auf 83,2\% unter Exemestan plus OFS, und zwar ohne zusätzliche Nebenwirkungen. In der TEXT-Studie betrug die Rate 81,6\% unter Exemestan und 79,2\% unter Tamoxifen, jeweils kombiniert mit einer OFS. Für eine endgültige Bewertung des OFS-Effekts sei es bei einem Follow-up von median bisher 5-6 Jahren noch zu früh, so die Forscher. Symptome, die die Lebensqualität von Teilneh- merinnen unter 35 am stärksten beeinträchtigten, waren vasomotorischer Art.

Fazit: Chemotherapeutisch behandelte Frauen unter 35 mit HR-positivem Brustkrebs scheinen von einer OFS in Kombination mit Tamoxifen oder Exemestan mehr zu profitieren als von $\mathrm{Ta}-$ moxifen allein. Die unerwünschten Wirkungen sind dabei offenbar nicht ausgeprägter als bei älteren Frauen vor der Menopause.

Peter Leiner

Saha P et al. Treatment Efficacy, Adherence, and Quality of Life Among Women Younger Than 35 Years in the International Breast Cancer Study Group TEXT and SOFT Adjuvant Endocrine Therapy Trials. J Clin Oncol. 2017;35(27):3113-22.

\title{
Laparoskopische Hysterektomie beim Endometriumkarzinom
}

\section{Die Standardtherapie beim Endometriumkarzinom ist die Entfernung des Uterus gepaart mit einer bilateralen Salpingo-Oophorektomie. Ist die totale laparoskopische Hysterektomie der totalen abdominalen ebenbürtig?}

$D^{i}$ ie kurzfristigen Vorteile, die die totale laparoskopische Hysterektomie (TLH) gegenüber der totalen abdominalen Hysterektomie (TAH) hat, begünstigten in jüngerer Vergangenheit einen Trend hin $\mathrm{zu}$ dem minimal-invasiven Eingriff. So ist die TLH etwa mit einer geringeren Morbidität verbunden. Um nun auch die Effizienz der beiden Techniken miteinander $\mathrm{zu}$ vergleichen, erhielten in der randomisierten Äquivalenzstudie LACE 760 Frauen (mittleres Alter 63 Jahre) eine TLH ( $\mathrm{n}=407)$ oder TAH $(\mathrm{n}=353)$. Alle waren an endometrioidem Endometriumkarzinom im Stadium I erkrankt. Primärer Endpunkt war das krankheitsfreie Überleben (DFS), definiert als Intervall zwischen der Operation und dem ersten Rezidiv, inklusive Krankheitsprogress oder die Entwicklung eines neuen Primärtumors oder Tod.

Nach median 4,5 Jahren Follow-up lag die Rate für das DFS in der TAH-Gruppe bei $81,3 \%$ und in der TLH-Gruppe bei $81,6 \%$. Die Differenz betrug 0,3\% (mit Vorteil für TLH; 95\%-Konfidenzintervall [KI] $-5,5$ bis $6,1 \%$; $=0,007)$. Damit wurde die definierte Äquivalenzgrenze von $\leq 7 \%$ eingehalten, womit sich die TLH der TAH hinsichtlich des DFS als äquivalent erwies. Es gab keinen statistisch signifikanten Unterschied zwischen den Gruppen bezüglich eines Rezidivs (7,9\% in der TAH-Gruppe vs. $8,1 \%$; Risikodifferenz $0,2 \% ; \mathrm{p}=0,93)$ oder bezüglich des Gesamtüberlebens (OS; 6,8\% in der TAHGruppe vs. 7,4\%; Risikodifferenz 0,6\%; $\mathrm{p}=0,76)$. Prognosefaktoren für DFS und OS umfassten die Vorgeschichte an malignen Erkrankungen, ein höheres Lebensalter und ein höheres Tumorstadium, nicht aber die Operationsmethode.

Fazit: Bei der Behandlung des Endometriumkarzinoms im Stadium I sind TLH und TAH mit einem vergleichbaren DFS und OS nach 4,5 Jahren verbunden. Dieses Ergebnis unterstützt angesichts der geringeren Komplikationsrate und kürzeren Erholungszeiten den Einsatz der TLH.

Kathrin von Kieseritzky

Janda M et al. Effect of Total Laparoscopic Hysterectomy vs Total Abdominal Hysterectomy on Disease-Free Survival Among Women With Stage I Endometrial Cancer. A Randomized Clinical Trial. JAMA. 2017;317(12):1224-33. 